\title{
Amino Acid Imbalance in Atherosclerosis
}

\author{
Karavay $\mathrm{PA}^{1}$, Nefyodov $\mathrm{LI}^{2 *}$ and Karavay $\mathrm{NL}^{3}$ \\ ${ }^{1}$ Department of Cardiology, Belarus \\ ${ }^{2}$ Department of Biochemistry, Belarus \\ ${ }^{3}$ Department of Hyperbaric Oxygenation, Belarus
}

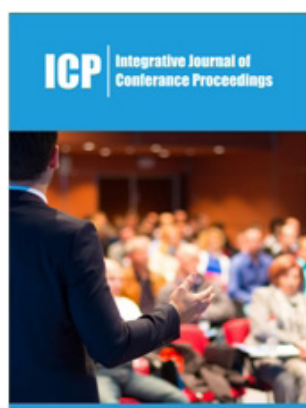

*Corresponding author: Nefyodov LI, Department of Biochemistry, Yanka Kupala Grodno State University, Belarus

Submission: 眥 January 20, 2020

Published: 酒January 29, 2020

Volume 2 - Issue 1

How to cite this article: Karavay P, Nefyodov L, Karavay N. Amino Acid Imbalance in Atherosclerosis. Int J Conf Proc.2(1). ICP.000530.2020.

Copyright@ Nefyodov LI, This article is distributed under the terms of the Creative Commons Attribution 4.0 International License, which permits unrestricted use and redistribution provided that the original author and source are credited.

\section{Abstract}

A review of literature data on the mechanisms of formation of the stock of free amino acids and their derivatives in atherosclerosis and methods for correcting metabolic imbalance.

Keywords: Amino acids, Derivatives, Atherosclerosis

\section{Introduction}

Our special interest in the study of the role of amino acids in the pathogenesis, prevention and treatment of atherosclerosis and its accompanying cardiovascular pathology in circulatory failure is due to several reasons. So, atherosclerosis is characterized not only by disturbances in the metabolic flows of carbohydrate (inhibition of gluconeogenesis and activation of glycolysis), lipid (activation of lipolysis, ketosis), but also protein (proteinand hypoalbuminemia), hyperglobulinemia and negative nitrogen balance) [1-18]. There is comparatively little data in the available literature on changes in the spectrum (pool, fund) of free plasma amino acids against the background of atherosclerosis. Only a few works are devoted to a comparative assessment and interpretation of changes in the pool of free amino acids at different stages of atherosclerosis and in the dynamics of its treatment $[12,19]$. The question of the information content of the established changes in the levels of individual amino acids in atherosclerosis and their significance in comparison with other clinical and biochemical criteria remains practically unclear. The unresolved problem of the choice of individual amino acids in the amino acids used for the directed correction of metabolic imbalance in atherosclerosis.

The importance of amino acids in the regulation of the functions of pathological conditions (vasoaterogenesis, arterial thrombosis) of the cardiovascular system has been convincingly established in a number of studies. Repeatedly described is a decrease in blood lipid levels under the action of glycine and its derivatives, the positive effect of cysteine and aspartate in patients with hyperlipidemia, the lipid-lowering effect of arginine in plasma [20]. High concentrations of amino acids and their derivatives in platelets have been demonstrated [14], upon activation of which the agonist binds to a specific receptor, forming a complex through which an energy signal that activates phosphatase and mobilizes ionized calcium from the dense tubular system to the cytoplasm is transmitted [21]. A study of the amino acid sequences of glycoprotein receptor polypeptides that specifically bind hem coagulation substrates showed the possibility of inhibiting platelet aggregation, adhesion and blood clot formation using synthetic and natural (snake venom) polypeptides containing arginine, glycine, asparagine, valine, proline, phenylalanine and cysteine [22]. The role of free amino acids in the processes of tissue ischemia tolerance and post ischemic recovery deserves special attention [5]. The protective effect of branched chain amino acids - BCAA (valine, leucine, and isoleucine) in the myocardium is manifested in the maintenance of contractility, macro erg levels (ATP, creatinine phosphate), normalization of aortic and coronary blood flow, cardiac output and cardiac output. The BCAA activates the production of catabolites of the adenine system during post ischemic reperfusion (65), activates the utilization of introduced amino 
acids to high-energy substrates of Krebs cycle, and helps to restore the functional capabilities of smooth muscle structures [23].

Recently, the role of amino acid derivatives, biogenic amines, in the development of the pathology of the cardiovascular system, the progression of thrombosis and damage to the vascular wall with the simultaneous activation of platelet function has been proven [24]. An increase in the content of adrenaline and norepinephrine in the blood, together with an increase in the content of other vasoconstrictor biogenic amines in the blood of patients with atherosclerosis, has been established at an evidence-based level. The predominance of $\alpha$-adrenergic receptors in arterial blood creates the conditions for the occurrence of vasoconstriction [25]. Thus, in patients with atherosclerosis, the activity of amino oxidase in the blood utilizing biogenic amines (serotonin, tyramine, and tryptamine) is significantly reduced, which creates the conditions for the manifestation of vascular effects of vasoconstrictor amines [26]. Platelet dense granules contain $\mathrm{Ca}_{2}+$, serotonin and other biogenic amines, ADP and ATP, secreted in the release reaction. $\mathrm{Ca}_{2}+$ ions are triggering factors in the formation of a platelet clot, spasm of blood vessels and acceleration of blood coagulation. Biogenic amines induce only primary aggregation [27]. The use of serotonin antagonists and the activation of catabolism enzymes of vasoconstrictor biogenic amines to eliminate the spastic component of ischemia seems to be a promising direction for the pathogenetic treatment of atherosclerosis. Thus, the functioning of the cardiovascular system is carried out according to the neuro-humoral regulatory mechanism, and the development of pathological conditions occurs with the involvement of various types of metabolism and cellular structures .

The adhesion-aggregation activity of platelets has a significant effect on the occurrence of thrombosis. As a result of platelet adhesion and aggregation, hemostasis is realized in small vessels. The emergence of adhesion is promoted by a change in the vascular wall, contact of platelets with collagen fibers, the release of ADP, biogenic amines, and traces of thrombin from damaged cells. Against the background of adhesion, platelet aggregation occurs, which is stimulated by ADP released from the formed elements of the blood as a result of the destabilization of their membranes under the action of prothrombinase. Platelet activation is a key step in the haemostatic process. Therefore, platelet activation is an essential stage of thermogenesis and vascular lesions [27,28].The antiatherogenic properties of the derivative of sulfur-containing amino acids Tau may be due to the fact that the synthesis of taurocholates promotes lipid absorption, lipolysis, and absorption of fatty acids in the intestine. On the other hand, conjugation of taurine (Tau) with bile acids affects the elimination of cholesterol from the body and thereby controls cholesterogenesis [12]. When rats are kept on a high-fat diet supplemented with Tau, the latter inhibits the rise in cholesterol in the liver, inhibiting its intestinal absorption. In addition, at a dose of 250 and $500 \mathrm{mg} / \mathrm{kg}$ body weight, Tau activated the transport of cholesterol from the blood and its metabolism to bile acids [12].

Adding 300-500mg of Tau to the diet reduces the concentration of bile acids and cholesterol in monkey bile and enhances the synthesis of taurocholates in piglets [14]. It is possible that the high level of taurocholates in some species of mammals (rats) complicates the modeling of experimental atherosclerosis, because the rate of exchange of bile acids increases due to the formation of chlotaurin. The anti-atherogenic effect of S-adenosylmethionine, evaluated by increasing the level of glutathione and improving macro-and microcirculation, was investigated against the background of the atherogenic effect of cholesterol (60). S-adenosylmethionine is recommended as an additive in amino acid mixtures for parenteral nutrition. Currently, the best-known commercial preparations of S-adenosylmethionine in Europe are Samyr, Samet and Gambrel. Summarizing the above, it should be noted that the use of amino acid preparations for atherosclerosis is rational and the strategy for their use should be based on the elimination of the amino acid imbalance present in this disease, and the correction of the free sulfur-containing amino acids stock, including the use of taurine, whose anti-atherogenic properties should be considered quite promising [1-18].

Recently, new evidence has been obtained of the participation of amino acids in the pathogenesis of atherosclerosis. Thus, data were obtained on changes in extracellular levels of neurotransmitter amino acids during atherosclerotic brain damage-an increase in the concentration of both excitatory (glutamate, aspartate) and inhibitory amino acids (GABA and taurine) compared with the control [28]. It should be borne in mind that amino acids are not only important precursors for the synthesis of proteins and other $\mathrm{N}$-containing compounds, but also participate in the regulation of the main metabolic pathways. For example, glutamate and aspartate are components of the malate/aspartate shunt, and their concentrations control the rate of mitochondrial oxidation of glycolytic NADH. Glutamate also controls the rate of urea synthesis not only as a precursor to ammonia and aspartate, but as a substrate for the synthesis of $\mathrm{N}$-acetyl glutamate, a significant activator of carbonyl phosphate synthase. This mechanism allows the regulation of urea synthesis at a relatively constant concentration.

Certain amino acids (leucine) stimulate protein synthesis and inhibit autophagy degradation of the protein regardless of changes in cell volume, since they stimulate motor and protein kinase, which is one of the components of signal transduction of insulin. In the case of low energy supply of cells, motor stimulation with amino acids is inhibited by activation of camp-dependent protein kinase. Amino acid-dependent signaling also promotes insulin production by $\beta$-cells. This stimulates the anabolic effect of amino acids [12]. It is well known that the heart is "metabolically omnivorous" because it is able to actively oxidize fatty acids, glucose, ketone bodies, pyruvate, lactate, amino acids and even its structural proteins (in decreasing order of preference). The energy of these substrates provides not only mechanical contraction, but also the operation of various Tran's membrane pumps and conveyors necessary to maintain ionic homeostasis, electrical activity, metabolism and myocardial catabolism. Cardiac ischemia and the resulting coronary and heart failure alter both the electrical and metabolic activity of the myocardium. The preference for substrates has been little studied, although hypoxia during ischemia significantly changes the relative 
selectivity of the heart in the use of different substrates. Metabolic changes in heart rhythm disturbance are the main component of cardiac myopathies. At the same time, the potential contribution of amino acids to maintaining the electrical conductivity of the heart and stability during ischemia is underestimated. Despite the obvious evidence that amino acids have a cardio protective effect in ischemia and other cardiac disorders, their role in the metabolism of the ischemic heart has not yet been fully elucidated [29-31].

Studies on the determination of taurine and a number of amino acids predominant in the myocardium (glutamate, aspartate, glutamine and asparagine) in coronary insufficiency showed their differences in the content in the left and right ventricles in coronary insufficiency. A comparison of the levels of these amino acids in aortic stenosis and coronary heart disease in myocardial biopsy specimens showed higher concentrations of taurine in the left ventricle in both situations [32]. With severe, progressive cardio sclerosis in the rabbit myocardium, the content of phenylalanine and tyrosine increased, which was also found in patients with coronary heart disease, and the degree of increase in the level of amino acids changed depending on the clinical forms of coronary atherosclerosis (angina pectoris of various functional classes, myocardial infarction).Methionine is a key essential amino acid, a donor of methyl groups and sulfur. It takes an active part in the metabolism of carbohydrates, fats and amino acids, the activation of antioxidant and detoxifying systems. Methionine serves as an essential precursor of cysteine, glutathione, taurine and, as a cysteine precursor, is involved in the synthesis of insulin and coenzyme A.

Methylation processes (involving S-adenosylmethionine) are associated with gene expression, the functioning of the sympathoadrenal system, and the formation of choline and acetylcholine [32]. With regard to coronary heart disease, a special role is played by violations of the formation of methionine, leading to the accumulation in the blood and urine of its predecessor, homocysteine. Examination and treatment of patients with homocysteinuria revealed early and active development of atherosclerosis in young patients: hyperhomocysts(e) anemia is a significant risk factor for the development of atherosclerosis and coronary heart disease. Clinical studies have revealed a significant effect of methionine on the growth of smooth muscle cells, followed by vascular endothelial dysfunction and the development of arterial hypertension with a high risk of thrombosis. It has been established that elevated homocysteine levels can be reduced by adding folic acid and B vitamins to food products. High plasma homocysteine levels during myocardial ischemia accelerate the oxidation of low and very low-density lipoproteins, thereby enhancing the development of atherosclerosis, altering the coagulation cascade and increasing thrombogenicity of blood decreases. Homocysteine with the participation of homocysteine thiolactone has in vitro and in vivo direct damaging effects on endothelial cells, leading to impaired endothelial vasodilation factor. Elevated homocysteine levels enhance lipid peroxidation through the generation of hydrogen peroxide and superoxide radicals. In addition to these factors, an increase in homocysteine levels stimulates the growth of smooth muscle cells in the vascular wall, exacerbating the narrowing of the bloodstream.

Lysine is involved in the formation of collagen, the strengthening of the vascular wall, in the formation of carnitine, promotes the utilization of fatty acids for the energy potential of cells and the preservation of the body's immune reactivity. When the artery walls rupture, the collagen threads connected to each other via lysine separate and protrude into the vascular lumen, like the lysine residues, and are washed by the circulating blood. Lipoprotein A, a specific form of cholesterol present in the bloodstream, has receptors for lysine, binds to it and penetrates the intima of blood vessels, thus starting the process of generating hydrogen peroxide and superoxide radicals. Arginine, a semi-essential amino acid, serves as a precursor to nitric oxide, which affects platelet aggregation and adhesive ability, reducing the ability to thrombosis and reducing the vascular reactivity of atherosclerotic altered arteries and promotes the formation of collagen in the walls of blood vessels [33].

Adding 11 essential amino acids to the diet of the elderly, including arginine in amounts of $11 \mathrm{~g}$. Within 2 weeks, it led to a decrease in the level of lipids, cholesterol, LDL and triglycerides in the blood plasma, exerting an anti-atherosclerotic effect [34]. It is known that the lipid content in blood plasma is controlled not only by the amount of dietary fats and carbohydrates, but also by dietary protein and amino acids. The source of such a protein is believed to be important. It is shown that, in addition, the amino acid and peptide composition of dietary protein is also of great importance. Sulfur-containing amino acids (SAA) are recognized as one of the most powerful modulators of lipid metabolism in a number of amino acids. SAA has been shown to act on HDL (high density lipoprotein) cholesterol and lower LDL (low density lipoprotein) lipoprotein. SAAs have some beneficial effects in atherosclerosis and related diseases (metabolic syndrome). The relative availability of SAA, as well as the amount of SAA in diet proteins, determine lipid metabolism. Although it is not completely clear how SAAs affect gene expression and lipid metabolism at the molecular level, it has been proven that SAAs act on metabolism through activation of transcription and post-translational modification of a number of regulatory proteins [35].

Taurine inhibits the development of atherosclerosis, has an anxiolytic effect, which is realized indirectly through its systemic effect on the synthesis of 5-HT and GABA. This amino acid has a depressant effect in the central nervous system, inhibiting neuronal activity in the spinal cord and brain [36]. Soy protein (hypo cholesterol emic effect), unlike casein with a hypercholesterolemia effect, contains a greater amount of arginine and glycine and causes an increase in the content of plasma arginine and glycine. Soy protein induces a low insulin/glucagon ratio in both patients with hypercholesterolemia and normocholesterolemic situations. Casein causes a high insulin/glucagon ratio among patients with hypercholesterolemia. Amino acids (arginine and glycine) induce a decrease, lysine and branched amino acids - an increase in serum cholesterol. It has been hypothesized that the control of cholesterol 
by insulin and glucagon is regulated by dietary and endogenous amino acids. The insulin / glucagon ratio is proposed as an early metabolic index of the influence of food proteins on serum cholesterol levels, a risk factor and the general mechanism by which nutrition factors influence the development of atherosclerosis and cardiovascular disease [37].

In the blood plasma of patients with endothelial disorders in atherosclerosis, the levels of citrate, GABA, glutamate and cysteine were significantly different in comparison with myocardial ischemia in the content of glutamate and phenylalanine. On this basis, a differential diagnosis of aortic damage with coronary heart disease is considered possible [38]. The human heart uses a large number of free amino acids) as regulators of both myocardial protein metabolism [39] and energy metabolism. The dependence of the myocardium on the amino acid fund of the heart increases with heart failure due to the high activity of the anabolic in the myocardium and the lack of energy for cardiomyocytes. Anabolic reactions in the heart are dependent on the oxidation of fatty acids and glucose. Normally, the functional activity of the Krebs cycle is significantly dependent on the concentration of amino acids. Free amino acids are stimulators of mitochondrial energy under anaerobic conditions, and also contribute to the substrate supply of cycle. Essential to the availability of heart amino acids is that their absorption by the myocardium depends solely on their arterial levels. The content of BCAA amino acids (branched chain) in myocardial metabolism is the most significant activator of anabolism in the heart, the level of which is not dependent on insulin. A slight increase in arterial amino acids leads to a significant increase in myocardial uptake. Amino acids play a crucial role in the metabolism of proteins and heart energy. In heart failure, the arterial pool of free AA, which is the determining factor in the absorption of amino acids by the myocardium, has not been practically studied. So, in comparison with the control, arterial amino acid levels were reduced in patients with heart failure. This decrease was associated with the severity of chronic heart failure and left ventricular dysfunction, in particular the level of aspartic acid [40].

A plant-based protein diet with a low Lys: Arg ratio has a beneficial normalizing effect on total serum cholesterol, LDL and adiponectin. To assess atherosclerosis, the Met: Gly ratio is also used. Amino acids as transceptors and regulators of nutrient fluxes by the mechanism of action on the metabolic fluxes of intracellular and extracellular concentrations of amino acids on motor proteins [41]. Amino acids are currently becoming increasingly widely regarded as cardio protective substrates that promote metabolism in the heart under anaerobic conditions and hypoxia through their participation in metabolic processes [29]. The specificity, ionic dependence, and kinetic properties of the amino acid transport systems identified in endothelial cells were compared and the regulation of the transport of vascular tone modulator by nitric oxide (NO) during hypoxia was studied. The transfer of L-arginine to endothelial and smooth muscle cells is modulated by atherogenic lipids. The joint localization of the cationic transporter of amino acids CAT-1, nitric oxide synthase (eNOS) in endothelial cells, which provides a new mechanism for regulating the formation of
NO when L-arginine enters the bloodstream, has been proven [42]. The "homocysteine" theory of arteriosclerosis was recognized when it was shown that after exogenous methionine loading, the concentration of homocysteine and cysteine was higher in patients with cardiovascular disease compared with a healthy control. Increased homocysteine levels have been demonstrated in coronary artery disease and atherosclerosis. It was found that in mice consuming a methionine-enriched diet a significant athermanous aortic pathology was observed even at normal plasma homocysteine levels [43].

The development and progression of atherosclerosis, which ultimately leads to cardiovascular disease, is causally associated with hypercholesterolemia. Mechanically, the interaction between lipids and the immune system during the progression of atherosclerotic plaques contributes to the chronic inflammation observed in the artery wall during atherosclerosis. Localized inflammation and increased intercellular interaction can affect the polarization and proliferation of immune cells through changes in amino acid metabolism. In particular, the amino acids L-arginine (Arg), L-homoarginine (hArg) and L-tryptophane (Trp) have been extensively studied in the context of cardiovascular diseases, and their effect has been established as key regulators of vascular homeostasis, similar to the functions of immune cells. Cyclic effects between endothelial cells, congenital and adaptive immune cells occur with a change in the metabolism of Arg, hArg and Trp, which have a significant effect on the development of atherosclerosis. Thus, the metabolism and biological functions of Arg, L-homoarginine, and Trp allow them to be reasonably used for the treatment of atherosclerosis [44,45].

\section{References}

1. Singh RB, Mengi SA, Xu YJ, Arneja AS, Dhalla NS (2002) Pathogenesis of atherosclerosis: A multifactorial process. Exp Clin Cardiol 7(1): 40-53.

2. Nefyodov L (1996) Amino acids and their derivatives (chemistry, biochemistry, pharmacology, medicine) Proc of Internat Symp, Grodno p. 124.

3. Nefyodov LI, Karavay PA, Karavay NL (2014) Regulatory action of free amino acids and development on the basis of highly of substances infusion solutions with pathogenetic deterministic composition. Expert Opin Environ Biol 3(3): 111-115.

4. Holden JT (1962) Amino acid pools. Elsevier, Amsterdam, Netherlands, p. 815.

5. Lubec C, Rosenthal GA (1990) Amino acids (chemistry, biology, medicine). Springer, Switzerland, p. 1196.

6. Nefyodov LI (2010) The results of biochemical research and development of nitrogen-containing compounds of natural origin: Methodology of exploitation of biological properties as universal natural regulators of metabolism and drugs.

7. Nefyodov LI (1999) Biological activity and transport of drugs .Proc of Internat Symp, Grodno, p. 189.

8. Nefyodov LI (2000) VI Ordinary general assembly society of biochemistry of Belarus. Proc of Internat Symp Grodno, p. 225.

9. Nefyodov LI (2001) Amino acids and their derivatives in biology and medicine. Proc of Internat Symp Grodno, p. 124.

10. Nefyodov LI (2001) Target - oriented regulation of metabolic equilibrium by amino acids and strategy of their application as drugs with directional 
effects, p. 327.

11. Fafournoux P, Bruhat A, Jousse C (2000) Amino acid regulation of gene expression. Bio Chem J 351(pt 1): 1-12.

12. Meijer A (2003) Amino acids as regulators and components of nonproteinogenic pathways. J Nutr 133(6 suppl 1): 2057S-2062S.

13. Bruhat A, Cherasse Y, Chaveroux C, Maurin AC, Jousse C, et al. (2009) Amino acids as regulators of gene expression in mammals: Molecular mechanisms. Biofactors 35(3): 249-257.

14. Karavay P, Nefyodov LI, Karavay NL (2016) Amino acids in metabolomics: Perspective for the use of regulatory effects of fee amino acids in the creation on their basis of infusion solutions. International Journal of Hematology \& Therapy 2(2): 1-2.

15. Nefyodov LI (1992) DSci dissertation abstract (biochemistry) "Formation of the stock of free amino acids and their derivatives under conditions of metabolic imbalance". Institute of Radiobiology of the Academy of Sciences of Belarus, Minsk, p. 32.

16. Nefyodov LI (1998) Mechanisms of regulatory effects and strategy of using amino acids and their derivatives as effective agents for metabolic therapy and medicinal preparations. Proc of Internat Symp Biological activity and Transport of Drugs, p. 189.

17. Nefyodov L(1998) Mechanisms of regulation of metabolic balance: Results and prospects for the use of amino acids and their compositions as universal biologically active natural regulators of directional action and effective drugs. Vests NAS Belarus Ser Bial Navuk 4: 61-69.

18. Nefyodov LI (2010) The results of biochemical research and development of nitrogen-containing compounds of natural origin: methodology of exploitation of biological properties as universal natural regulators of metabolism and drugs.

19. Nefyodov L (1995) Taurine (biochemistry, pharmacology, medical application) Grodno RIPH, p. 145.

20. Nefyodov LI (1999) Biological activity and transport of drugs. Proc of Internat Symp Grodno, p. 189.

21. Young Lee, Eun-Hee Kim (2019) Therapeutic effects of amino acids in liver diseases: Current studies and future perspectives. J Cancer Prev 24(2): 72-78.

22. Wu G (2013) Functional amino acids in nutrition and health. Amino Acids 45(3): 407-411.

23. Bender DA (1975) Amino acid metabolism, Willey, USA p. 234.

24. Wu G, Wu Z, Dai Z, Yang Y, Wang W, et al. (2013) Dietary requirements of "nutritionally non-essential amino acids" by animals and humans. Amino Acids 44(4): 1107-1113.

25. Nefyodov LI, Klimovich II, Moroz AR (1991) Statistical analysis of amino acid pool structure in donors blood plasma. Zdravoochranenie Belarusi 11: 10-13.

26. Nefyodov LI (1992) Biological activity of taurine (review). Vesti AN BSSR 3-4: 99-106.

27. Nefyodov LI (1990) Taurine metabolism in mammals (review). Vesti AN BSSR 5: 99-106.

28. Patel RP, Levonen A, Crawford JH, Darley U (2000) Mechanisms of the pro- and anti-oxidant actions of nitric oxide in atherosclerosis. Cardiovasc Res 47: 465-474.
29. Drake KJ, Sidorov VY, Guinness OP, Wasserman DH, Wikswo JP (2012) Amino Acids as metabolic substrates during cardiac ischemia. Exp Biol Med (Maywood) 237(12): 1369-1378.

30. Zalba G, Beaumont J, Jose G, Fortuno A, Fortuno MA, et al. (2000) Vascular oxidant stress: molecular mechanisms and patho-physiological implications. J Physiol Biochem 56(1): 57-64.

31. Aziz M, Yadav KS (2016) Pathogenesis of Atherosclerosis. Med Clin Rev 2(3): 1-6.

32. Martin Lewis , Littlejohns, Hua Lin, Angelini GD, Suleiman MS (2014) Cardiac taurine and principal amino acids in right and left ventricles of patients with either aortic valve stenosis or coronary artery disease: the importance of diabetes and gender. Springer Plus 3(1): 1-11.

33. Brosnan JT (2001) Amino acids, then and now: a reflection on Sir Hans Krebs contribution to nitrogen metabolism. IUBMB Life 52(6): 265-270.

34. Borsheim E, Bui QU, Tissier S, Cree MG, Ronsen O, et al. (2009) Amino acid supplementation decreases plasma and liver triglycerides in elderly. Nutrition 25(3): 281-288.

35. Hiroaki Oda (2006) Functions of sulfur-containing amino acids in lipid metabolism. The Journal of Nutrition 136(6): 1666S-1669S.

36. Kothandam H, Biradugadda P, Maganti B, Keerthi T, Vegunta B, et al. (2012) Taurine, "A key amino acid in the drug discovery"-a review. Asian Journal of Biomed \& Pharmaceut Sciences 2(12): 21-27.

37. Sanchez, Hubbard RW (1991) Plasma amino acids and the insulin/ glucagon ratio as an explanation for the dietary protein modulation of atherosclerosis. Medical hypothesis 35(4): 324-329.

38. Linlin Wang, Sha Liu, Wengang Yang, Haitao Yu, Li Zhang, et al. (2017) Plasma amino acid profile in patients with aortic dissection. Scientific Reports 7: 1-9.

39. Aquilani R, Rovere MT, Corbellini D, Pasini E, Verri M, et al. (2017) plasma amino acid abnormalities in chronic heart failure. Mechanisms, potential risks and targets in human myocardium metabolism. Nutrients 9(11): 1251.

40. Ma LL, Ji GY, Jiang ZQ (2014) Influence of dietary amino acid profile on serum lipids in hypercholesterolemic chinese adults. J Nutr Food Sci 4(1): 1-6.

41. Milan Holeček (2018) Branched-chain amino acids in health and disease: metabolism, alterations in blood plasma, and as supplements. Nutrition \& Metabolism15: 1-12.

42. Giovanni E, Yudilevich DL, Sobrevia L (2003) Regulation of amino acid and glucose transporters in endothelial and smooth muscle cells. Physiol Rev 83(1): 183-252.

43. Selhub J, TroenAM (2016) Sulfur amino acids and atherosclerosis: a role for excess dietary methionine. Ann N Y Acad Sci 1363(1): 18-25.

44. Nitz K, Lacy M (2019) Amino acids and their metabolism in atherosclerosis. Arterioscler Thromb Vasc Biol 39(3): 319-330.

45. Yanni AE, Agrogiannis G, Nomikos T, Fragopoulou E, Pantopoulou A, et al. (2010) Oral supplementation with L-aspartate and L-glutamate inhibits atherogenesis and fatty liver disease in cholesterol-fed rabbit. Amino Acids 38(5): 1323-1331. 